\title{
Changing life expectancy in the 1980s: why was Denmark different from Sweden?
}

Health Services

Research Unit, London School of Hygiene and tropical Medicine, Keppel Street, London WC1E 7HT

L Chenet

M McKee

Department of Social Medicine, University of Copenhagen, Panum Institute, Blegdamsvej 3, 2200N, Denmark

M Osler

A Krasnik

Correspondence to: Dr M Osler

Accepted for publication January 1996

\begin{abstract}
Objective - To identify the contribution from specific causes of death to the changes in life expectancy at birth in Denmark relative to Sweden in different age groups during the 1980 s and to compare the difference in life expectancy between the two countries in 1990.

Design - Mortality data from WHO mortality tapes grouped in smaller series of clinically meaningful categories were used to calculate the contribution of each of these categories at each 10 year age group to the difference in life expectancy at birth in each country between 1979 and 1990 and between the two countries.

Setting - Denmark and Sweden.

Results - Between 1979 and 1990 life expectancy increased in both Denmark and Sweden. However, the increase in Sweden was more than two years while that in Denmark was less than one year. In both countries a decrease in cardiovascular disease mortality contributed most to the increase in life expectancy in males as well as females. In both sexes the smaller increase in life expectancy in Denmark was a result of differences in mortality trends in cardiovascular diseases and respiratory and non-respiratory cancers.

Conclusion - Over a short time two Nordic countries experienced remarkable but different changes in mortality. These findings suggest that mortality rates are sensitive to even minor differences in social and cultural factors across countries and over short time periods.
\end{abstract}

(F Epidemiol Community Health 1996;50:404-407)

Among the countries of western Europe, Denmark had the smallest improvement in life expectancy during the 1980 s. $^{1}$ This difference is especially noticeable when Denmark is contrasted with its neighbouring countries. While Sweden experienced an increase in male life expectancy at birth of 2.6 years between 1979 and 1990 , the corresponding figure for Denmark was only 0.9 years. The corresponding figures for females were 2.0 and 0.35 , respectively.

In 1993 the Danish Ministry of Health set up a committee to examine possible explanations for the stagnating life expectancy in Denmark. The main conclusion of this investiga- tion was that the stagnation could not be due to poorer treatment of diseases, but to failed prevention. $^{2}$

The present study aimed to identify the contributions specific causes of death made to the changes in life expectancy at birth in Denmark relative to Sweden in different age groups during the $1980 \mathrm{~s}$ and to compare the difference in life expectancy between the two countries in 1990. We used the statistical approach developed by Pollard, ${ }^{34}$ which offers an alternative to the traditional measures of general life expectancy and changes in cause specific death rates by examining how the latter contribute to the overall changes in life expectancy and the effect of changes at each age group. By identifying differences in mortality trends between countries that are socially and culturally alike, it is possible to develop new hypotheses on factors responsible for population mortality.

\section{Methods}

We analysed mortality data from the World Health Organization (WHO) mortality tapes, classified according to the abbreviated 8th (Denmark, 1979 and 1990 and Sweden 1979) and 9th (Sweden 1990) revisions of the International Classification of Diseases (ICD), for Denmark and Sweden for the years 1979 and 1990.

Life tables for both sexes were constructed using standard techniques. The probabilities of death from any cause in each age band in the two countries were compared.

The abbreviated causes of death were grouped to create a smaller series of clinically useful categories (table 1). The selection was based on the numbers of deaths and the association with known risk factors. The categories were also selected in such a way as to minimise the effects of the change in coding in Sweden during this period. The contributions each of these categories at each 10 year age group (the under 1 and 1 to 4 age groups were analysed separately) made to the difference in life expectancy at birth in each country between 1979 and 1990 and between the two countries were calculated using the approach developed by Pollard. ${ }^{34}$ Designating life expectancy at birth for populations 1 and 2 as $e_{0}$ and $\mathrm{e}_{0}{ }^{2}$, the difference between the two life expectancies can be written:

$$
\begin{gathered}
\mathrm{e}_{0}^{1-}-\mathrm{e}_{0}^{2}=\sum \mathrm{n}\left({ }_{n} \mathrm{~m}_{\times}{ }^{(\mathrm{i}) 1}{ }_{\mathrm{n}} \mathrm{m}_{\times}{ }^{(\mathrm{i}) 2}\right) \cdot \mathrm{w}_{\times} \\
\mathrm{w}_{\times}=\frac{1}{2}\left({ }_{\times} \mathrm{p}_{0}^{2} \mathrm{e}_{\times}^{1}{ }_{\times}{ }_{\times} \mathrm{p}_{0}^{1} \mathrm{e}_{\times}^{2}\right)
\end{gathered}
$$

where ${ }_{n} m_{x}(i)$ is the central mortality rate for cause $i$ between age $x$ and $x+n$. 
Table 1 Constituents of disease categories used in the study

\begin{tabular}{lll}
\hline Disease group & Abbreviated ICD8 & Abbreviated ICD9 \\
\hline Infectious diseases & $000-136$ & $001-138$ \\
Respiratory cancer & $161-162$ & $161-162$ \\
Breast cancer & 174 & 174 \\
Other neoplasms & $140-154,170-173,180-239$ & $140-157,170-173,179-239$ \\
Cardiovascular disease & $390-429,440-458$ & $390-429,440-459$ \\
Cerebrovascular disease & $430-438$ & $430-438$ \\
Respiratory disease & $460-519$ & $460-519$ \\
Genitourinary disease, & $580-678$ & $580-629$ \\
$\quad$ obstetrics & & \\
Congenital/perinatal diseases & $741-779$ & $740-799$ \\
Suicide & E950-959 & E950-959 \\
Accidents & E810-949, E960-999 & $5800-949$, E960-999 \\
Cirrhosis & 571 & 571 \\
Other diseases & $240-389,520-560,572-577,240-285,290-319,320-389$, & $520-529,530-560,574-579$, \\
& $680-734,780-795$ & $680-736,780-799.1$ \\
\hline
\end{tabular}

* Heart and vascular diseases, excluding cerebrovascular diseases

The weight $\mathrm{w}_{\mathrm{x}}$ is given by the formulae:

where ${ }_{x} p_{0}$ is the life table probability of surviving from birth to age $x$ and $e_{x}$ the life expectancy at age $x$, that is the mean numbers of years left.

This could also be written as:

$$
\mathrm{e}_{0}^{2}-\mathrm{e}_{0}^{1}=\sum_{\times} \sum_{\mathrm{i}}\left(\mathrm{Q}_{\times}^{(\mathrm{i}) 1}-\mathrm{Q}_{\times}{ }^{(\mathrm{i}) 2}\right) \mathrm{w}_{\times}
$$

with:

$$
Q_{x}=-\ln \left(\frac{1_{x+n}}{1_{x}}\right)
$$

where $l_{x}$ is the life table number of people alive at age exactly $x$.

The quantities

$$
\left(Q_{\times}^{(i) 1}-Q_{\times}{ }^{(i) 2}\right) w_{\times}
$$

give the weight of each cause in the difference observed between the two life expectancies.

The sum over all ages gives the total contribution for each cause $i$ in that difference while the sum over causes would give the relative weight of mortality at each age $\mathbf{x}$.

\section{Results}

Table 2 shows the age standardised mortality rates in 1979 and 1990 and the contributions to changes in life expectancy of the specific causes in males and females in Denmark and Sweden. (Detailed data on changes in life expectancy in the different age groups are available from the authors. ${ }^{\star}$ ) For Danish males, changes in deaths from cardiovascular diseases contributed almost one year to the increase in life expectancy during the study period (table 2). The other main positive contributions among Danish males were made by the changes in the number of deaths from accidents and congenital/perinatal disorders. These were counterbalanced, in part, by negative changes in deaths from infectious diseases, non-respiratory malignant diseases, and "other diseases". When analysed by age group, changes in deaths in the ages 25 to 44 made a net negative contribution (data available from authors $\left.{ }^{\star}\right)$. There was a relative large positive contribution from reductions in deaths from accidents in the 15 to 24 years age group.

For Swedish males, changes in deaths in all age groups and from all causes except "other diseases" made a positive contribution to the increase in life expectancy over this period (table 2). The greatest contribution was made by deaths from cardiovascular diseases - this accounted for almost 1.4 years, much more than in Denmark. The second largest contribution was made by deaths from accidents accounting for almost 0.4 of a year, which was twice as high as in Denmark.

For Danish females, deaths in the age groups 5 to 14 and 55 to 74 made a negative contribution to the change in life expectancy (data available from the authors ${ }^{\star}$ ). When analysed by cause, negative contributions were made by deaths from respiratory cancer, other respiratory diseases, and "other diseases", with further very small negative contributions from infectious diseases and cirrhosis. The largest positive contribution was, as with males, from deaths from cardiovascular diseases.

For Swedish females too, the largest positive contribution to the change in life expectancy was made by deaths from cardiovascular diseases and, as with males, this was much larger than in Denmark (table 2). Unlike the situation with Swedish males, but like that with females in Denmark, deaths from respiratory

Table 2 Cause specific, age standardised mortality rates per 100000 (SDR) in 1979 and 1990 and change in life expectancy (LE) in years among males and females in Denmark and Sweden

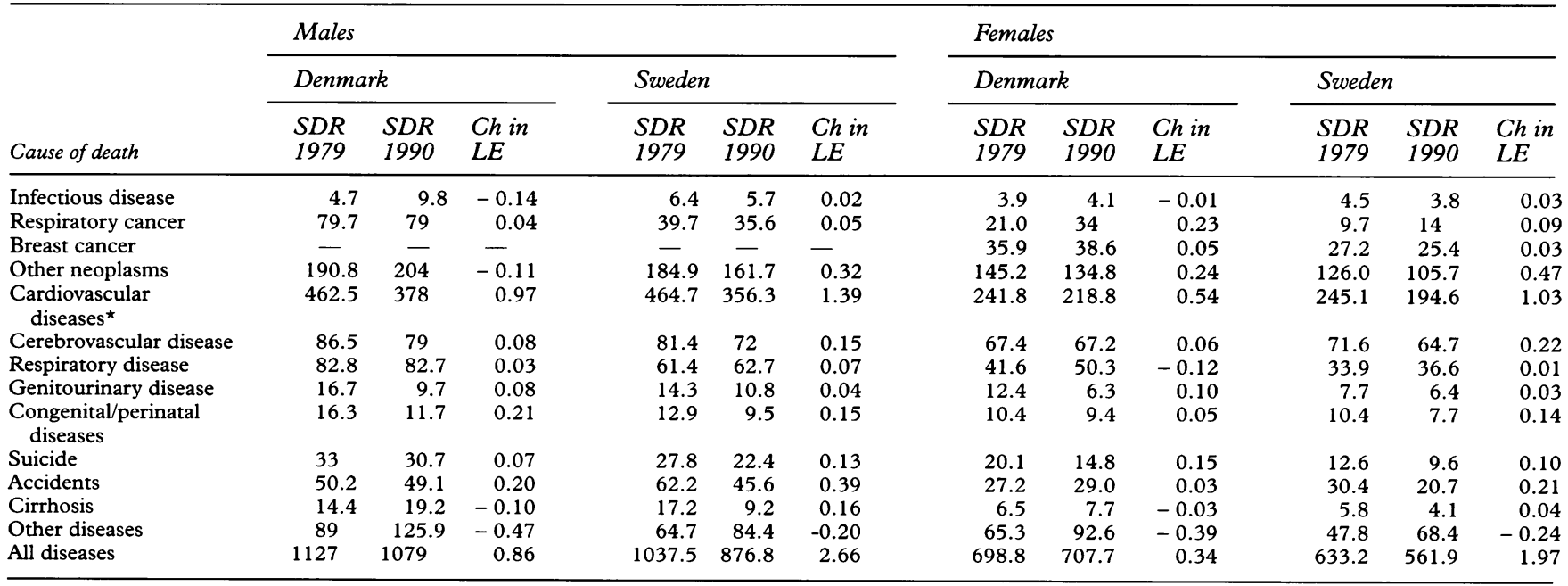

* Heart and vascular diseases excluding cerebrovascular diseases.

$\mathrm{Ch}$ in $\mathrm{LE}=$ change in life expectancy. 


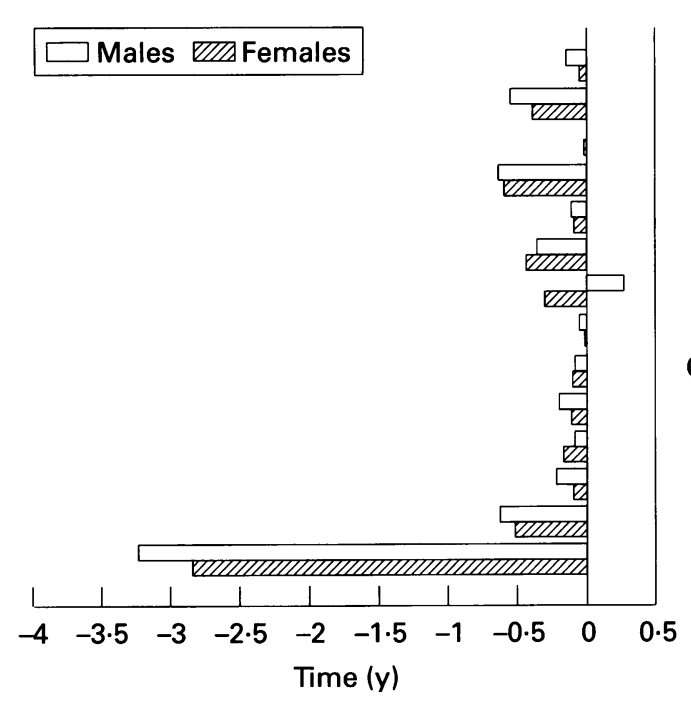

Infectious disease Respiratory cancer Breast cancer

Other neoplasms

Cardiovascular disease Cerebrovascular disease

Respiratory disease

Genito-urinary disease Congenit/perinatal disease Suicides Accidents Cirrhosis

Other diseases

All diseases

Figure 1 Contribution of leading causes of death to differences in life expectancy in years at birth between Denmark and Sweden, 1990.

cancer made a negative contribution. There was a large positive contribution from "other malignant diseases" in Swedish males and Danish females, unlike the negative contribution in Danish males.

While the preceding analysis indicates the consequences of changes over time, figure 1 shows the difference between the two countries in 1990. For males, deaths from nonrespiratory and respiratory cancers were responsible for the largest contribution to the difference, accounting for one third of the net difference. Deaths at all ages except 15 to 24 had a net effect unfavourable to Denmark (data available from the authors ${ }^{\star}$ ).

For females, the largest contribution to the different life expectancies in 1990 was made by deaths from non-respiratory cancers, followed by "other diseases" and respiratory cancers (fig1). Deaths at all ages except 25 to 34 had a net effect favouring Sweden (data available from the authors ${ }^{\star}$ ).

\section{Discussion}

Between 1979 and 1990, life expectancy increased in both Denmark and Sweden. However, Sweden experienced the greater increase (more than two years), while the increase in Denmark was less than one year. In both countries and in both sexes a decrease in cardiovascular disease mortality contributed most to the increase in life expectancy. However, in both males and females the less favourable development in Denmark was mainly a result of differences in mortality from cardiovascular diseases and respiratory and non-respiratory cancers.

The contributions of specific causes to the changes over time in relation to the differences between Denmark and Sweden could be artefactual or real. Artefactual figures may be the result of errors due to national changes or international differences in rules and procedures for classification of death or in diagnostic variation. Such changes that do exist between the two classifications, such as the revised rules for coding asthma or categorising rheumatic heart disease, either have no effect on the allocation of deaths to the categories used in this study or involve very few death. ${ }^{5}$ In addition, the main structures and functions of the Danish and Swedish health services and the basic training are similar and did not change significantly during the study period. This means that the likelihood of diagnostic variation is rather small.

Life expectancy does not measure death rates directly - rather it is an indicator of the consequences of period mortality effects over the hypothetical life time of individuals.

In order to develop hypotheses about the factors responsible for the identified changes and differences in life expectancy, we tried to compare the development in social conditions and lifestyle between Denmark and Sweden, using information from comparable health surveys and statistics. Superficially, there are many similarities between the two countries with regard to living conditions (for example, gross national product, social security transfers, and housing), ${ }^{6}$ although, as has been noted elsewhere recently, what appears to outsiders as heterogeneity within Scandinavia may be more varied on closer inspection. ${ }^{7}$ For the purposes in this study, the most striking feature was the timing of economic recession. This began in Denmark in 1986/87 but did not occur in Sweden until 1991/92, although unemployment was higher in Denmark throughout this period. The rate of unemployment was nearly constant at around $8 \%$ in Denmark and $2 \%$ in Sweden. ${ }^{8}$ There is a considerable body of evidence linking unemployment with ill health. ${ }^{9}$ It is also notable that fear of unemployment was strikingly higher in Denmark. In 1990, $28 \%$ of those in employment in Denmark were worried about unemployment whereas only $3 \%$ in Sweden had the same anxiety. ${ }^{8}$ This is important, given the evidence from a recent British study that the fear of unemployment is also associated with ill health. ${ }^{10}$ We are, however, unaware of any Scandinavian studies documenting this effect.

The reasons for the association between unemployment and health have been debated for many years. ${ }^{11}$ The principal theories are that the effect is an artefact or due to social selection or behavioural or materialist factors. It is now widely accepted that the first two are of minor importance but there is a countinuing debate about the relative importance of behavioural factors, based on the view that worse health among the poor can be explained by behaviour, such as higher rates of smoking, and the materialist theory that argues that the poverty in itself has an independent effect on health. The situation is complicated even further by differences in the access of populations to social support that may enable them to cope better with adversity. For example, evidence from the MONICA study shows that a higher percentage of Danish adults compared with Swedish adults live alone $(27 \%$ versus $18 \%$ ), suggesting that Danes have less well developed social networks than Swedes. ${ }^{12}{ }^{13}$

This study cannot disentangle this complex picture but the evidence we have gathered does at least provide the basis for more detailed comparative studies. There were substantial 
differences in lifestyle. ${ }^{12-15}$ Smoking, although declining in most countries, is still higher in Denmark among both men and women. In $1980,59 \%$ of men and $43 \%$ of women in Denmark were smokers. Ten years later the percentages had decreased by 10 percentage points to $49 \%$ and $41 \%$, respectively. During the same period the percentage who smoked in Sweden decreased from $39 \%$ to $27 \%$ in men and from $29 \%$ to $25 \%$ in women. Both alcohol and fat consumption are also higher in Denmark, although somewhat surprisingly, these findings are not reflected in body mass indicies and cholesterol concentrations, both of which are actually lower in Denmark. In the 1980 s, alcohol consumption in Denmark was 12 litres per capita and that in Sweden was 6 litres per capita. In $1988,45 \%$ of the energy in the Danish diet and $38 \%$ of that in the Swedish diet was derived from fat. The population studies have shown that the percentages of the adult population with a serum cholesterol concentration of more than $6.5 \mathrm{mmol} / \mathrm{l}$ were $33 \%$ in Denmark and $41 \%$ in Sweden, while $10 \%$ of Danish adults and $14 \%$ of Swedish adults had a body mass index over $29 \mathrm{~kg} / \mathrm{m}^{2}$.

Both countries have undertaken community intervention programmes, achieving small changes in risk factors. ${ }^{12}{ }^{16}$ However, the Swedish Government has tried to reduce alcohol and tobacco use by very restrictive legislation. In Sweden, adversing of tobacco and alcohol products is banned, but this is still, with some limitations, allowed in Denmark, and today tobacco advertising agressively targets women. Sweden introduced health warnings on tobacco products as early as 1977 , but this was not begun in Denmark until 1990. In addition, sales of wine and spirits in Sweden are limited to special stores ("Systembolag"), while in Denmark all supermarkets can sell stronger alcoholic beverages and, as it is a member of the EU, the prices of alcoholic beverages have fallen during the $1980 \mathrm{~s}$. It is therefore interesting to note that while the premature death rate from chronic liver disease actually increased in Denmark from 7.4 to $11.3 / 100.000$ reflecting an increase for both sexes, it fell considerably in both sexes in Sweden from 9.3 to 4.9 / 100.000 .

The differences in exposure to recognised factors, such as tobacco, alcohol, and dietary fat are consistent with the relatively worse Danish figures for disorders such as cardiovascular disease and cirrhosis. This analysis has shown, however, that deaths from a wide range of diseases contribute to the slower improvement in life expectancy in Denmark, suggesting that while there is scope for improvement through healthy public policies, as seen in Sweden, there may be more deep seated problems due to unemployment, financial insecurity, and access to social support.

In summary, during the 1980 s, two neighbouring countries that seem to be very similar experienced quite different health outcomes. This paper contributes to a better understanding of this difference but it also highlights the many gaps in our knowledge. It offers yet another example of the scope within Europe for learning from the experience of others. ${ }^{17}$

*Note: To obtain a copy of an appendix with age specific changes in life expectancy send a request to email: 1.chenet@lshtm.ac.uk.

Wilkinson RG. Income distribution and life expectancy. BMf 1992:304;165-8.

2 Ministry of Health. Life expectancy in Denmark. Copenhagen: Ministry of Health, 1994

3 Pollard $\mathrm{JH}$. The expectation of life and its relationship to mortality. fournal of the Institute of Actuaries 1982;9: 225-40.

4 Pollard JH. Cause of death and expectation of life: some international comparisons. In: Vallin J, D'Souza S, Palloni A, eds. Measurement and analysis of mortality: new approaches. Liege: Oxford University Press, 1987: 269-91. tics. Comparison of 8th and 9th revisions of the international tics. Comparison of 8th and 9th revisions of the international
classification of diseases. Series DHI no10. London: OPCS, 1983.

6 Organisation for Economic and Cooperation and Development. Economic outlook. Historical statistics 1960-1990. Paris: OECD, 1992.

7 McKee M. Seamless in Scandinavia. European Health Reform 1995;2:5.

8 Vogel J. Living in Norden. Living conditions and inequalities at the end of the 1980s. Copenhagen: Nordic Statistics the end of the

9 Smith R. Unemployment and health: $a$ disaster and challenge. Oxford: Oxford University Press, 1987

10 Ferrie JE, Shipley MJ, Marmot MG, Stansfield S, Davey Smith $G$. Health effects of anticipation of job change and non-employement longitudinal data from the Whitehal study. $B M \mathcal{F}$ 1995;311:1264-9.

11 Department of health and Social security. Inequalities in health: report of a research working group (The Black report). London:HMSO, 1980.

12 Brännström I, Weinehall L, Perrson LÅ, Wester PO, Wall S. Changing social patterns of risk factors for cardiovascular disease in a Swedish community. Int f Epidemiol 1993; 22:1026-37

13 Osler M. Social class and health behaviour in Danish adults. A longitudinal study. Public Health 1993;107:251-60.

14 Sjøl A, Schroll $M$. The cardiovascular risk profile in the Glostrup region. MONICA screening II 1986/87. Ugeskr Laeger 1990;152:3449-55.

15 World Health Organization. Health year 2000. WHOdatabase. Copenhagen: WHO Reginal Office, 1993.

16 Osler M, Jespsersen NB. The effect of a community-based cardiovascular disease prevention project in a Danish Municipality. Dan Med Bulletin 1993;40:485-9.

17 Abel-Smith B, Figueras I, Holland W, McKee M, Mossialos F. Choice in health policy: An agenda for the European Union. Aldershot \& Luxembourg: Dartmouth Press/ Office for Official Publications of the European Communities, 1995. 